\title{
Targeted Therapy After Brain Radiotherapy for BRAF-Mutated Melanoma With Extensive Ependymal Disease With Prolonged Survival: Case Report and Review of the Literature
}

OPEN ACCESS

Edited by:

Jethro Hu,

Cedars-Sinai Medical Center

United States

Reviewed by:

Sunit Das,

St. Michael's Hospital, Canada

Brad E. Zacharia,

Penn State Milton S. Hershey Medical

Center, United States

${ }^{*}$ Correspondence:

Jennifer S. Yu

yuj2@ccf.org

†Present Address:

Ibrahim Abu-Gheida,

Department of Radiation Oncology,

University of Texas MD Anderson

Cancer Center, Houston, TX, United States

Specialty section:

This article was submitted to Neuro-Oncology and Neurosurgical

Oncology,

a section of the journal

Frontiers in Oncology

Received: 05 September 2018 Accepted: 26 February 2019

Published: 26 March 2019

Citation:

Abu-Gheida I, Chao S, Murphy E, Suh J, Stevens GH, Mohammadi AM, MCNamara M and Yu JS (2019)

Targeted Therapy After Brain Radiotherapy for BRAF-Mutated

Melanoma With Extensive Ependymal

Disease With Prolonged Survival:

Case Report and Review of the

Literature. Front. Oncol. 9:168.

doi: 10.3389/fonc.2019.00168
Ibrahim Abu-Gheida ${ }^{1+}$, Samuel Chao ${ }^{1}$, Erin Murphy ${ }^{1}$, John Suh ${ }^{1}$, Glen H. Stevens ${ }^{2}$, Alireza M. Mohammadi ${ }^{3}$, Michael McNamara ${ }^{4}$ and Jennifer S. Yu ${ }^{1,5 *}$

1 Department of Radiation Oncology, Taussig Cancer Center Institute, Cleveland Clinic, Cleveland, OH, United States, ${ }^{2}$ Department of Neurology, Neurological Institute, Cleveland Clinic, Cleveland, $\mathrm{OH}$, United States, ${ }^{3}$ Department of Neurological Surgery, Neurological Institute, Cleveland Clinic, Cleveland, OH, United States, ${ }^{4}$ Department of Hematology and Medical Oncology, Taussig Cancer Center Institute, Cleveland Clinic, Cleveland, OH, United States, ${ }^{5}$ Department of Cancer Biology, Lerner Research Institute, Cleveland Clinic, Cleveland, OH, United States

Melanoma brain metastasis with ependymal spread/metastases is uncommon. These cases are frequently classified together with leptomeningeal disease. However, the commonalities and differences in the underlying pathophysiology and clinical outcomes between these two types of spread are not clear. Very few reports on long term outcome and durable central nervous system (CNS) disease control have been reported in the literature. Here, we report a case of a 45 year-old Caucasian lady with BRAF-V600E mutant metastatic melanoma to the brain who had whole brain radiotherapy followed by two Gamma knife radiosurgery treatments for localized disease progression. She then developed extensive ependymal disease progression with no evidence of leptomeningeal spread. She was treated with a repeat course of whole brain radiotherapy and maintained on BRAF and MEK inhibitors with durable CNS disease control for more than a year. This study reviews the management of BRAF-V600E mutant melanoma with ependymal involvement. Management using radiation therapy with maintenance targeted therapy seems to be a reasonable approach to this challenging disease.

Keywords: melanoma, ependymal disease, leptomeningeal disease, whole brain radiation, radiosurgery, BRAF inhibitor, MEK inhibitor, brain metastasis

\section{INTRODUCTION}

A 45 year-old Caucasian woman presented to our institution for management of brain metastasis. Four years prior to her presentation, she was diagnosed with left upper back BRAF V600E mutant melanoma. She underwent wide local excision with sentinel lymph node biopsy of the left axilla with no nodes positive. She did well for the next 3 years until she presented with an isolated biopsy-proven right axillary recurrence. She underwent right axillary lymph node dissection with pathology demonstrating one out of 16 lymph nodes positive, measuring $3.5 \mathrm{~cm}$ with extranodal extension. The patient then received adjuvant radiation to the right axilla followed by interferon treatment for 1 year. At the end of interferon treatment, surveillance PET/CT showed no evidence 
of extracranial systemic disease. However, MRI brain showed a solitary $1.6 \mathrm{~cm}$ left temporoparietal lesion (Figure 1). The patient was asymptomatic and was treated with whole brain radiation therapy (WBRT) at an outside hospital. Two months after completing radiation, the patient developed dizziness and had an episode of generalized tonic clonic seizure. MRI brain showed progression of the left temporoparietal lesion. She was referred to our center for further treatment and was subsequently treated by Gamma Knife radiosurgery (GKRS). Follow up MRI showed that the treated lesion was stable, but there was an additional enhancing lesion in the left inferomedial cerebellum. This lesion was asymptomatic. The patient also received GKRS to the cerebellar lesion with excellent control. Serial brain MRIs and MRI perfusion over the next 6 months showed no new lesions. The patient developed radiographic evidence of radiation necrosis in the left parietal lobe and was maintained on steroids with good response. However, she developed side effects from steroids including proximal muscle weakness and significant weight gain that interfered with her functioning. Attempts to wean her off steroids were unsuccessful. She was being evaluated for laser ablation to reduce cerebral edema associated with her radiation necrosis. On workup for this procedure, she was found to have progression of brain metastases including a new $3 \mathrm{~mm}$ enhancing focus involving the right anterior body of corpus callosum and enhancing, linear and nodular lesions along the ventricles consistent with ependymal spread (Figure 2A). Complete spine MR imaging showed no evidence of gross spinal leptomeningeal disease. Considering her extensive brain disease, she was not a candidate for laser ablation. She received a second course of WBRT. She then received dual BRAF/MEK inhibition with dabrafenib and trametinib for 1 year. MRI 2 months post-WBRT showed a decrease in ependymal enhancement; the previously treated metastasis was stable (Figure 2B). In the meantime, multiple failed attempts were made to wean the patient off of steroids. The patient suffered from steroid related side effects including weight gain, diabetes, and proximal muscle atrophy and was switched to bevacizumab for radiation necrosis. Bevacizumab was discontinued after the patient developed gastrointestinal bleeding. During this time, she continued to be maintained on dabrafenib and trametinib. Her last follow up brain MRI showed slight increase size of the left parietal radiation necrosis, but her ependymal disease was stable and there were no new lesions (Figure 2C). Within 1 month, however, the patient developed widespread disease. She enrolled in hospice care and passed away 1 week later.

\section{BACKGROUND}

The incidence of melanoma has been rising for the last 30 years. It is estimated that $\sim 76,000$ individuals will be diagnosed with melanoma and about 10,000 will succumb to their disease (1). Being the third most common brain metastasis diagnosed after lung and breast cancer, melanoma has the highest risk of spread to the CNS among all common cancer types with a $40-60 \%$ chance of patients developing brain metastases at some point in the course of their disease. Autopsy series identified CNS involvement in up to $80 \%$ of patients with metastatic melanoma (2). Several studies indicate a median survival of $\sim 4$ months

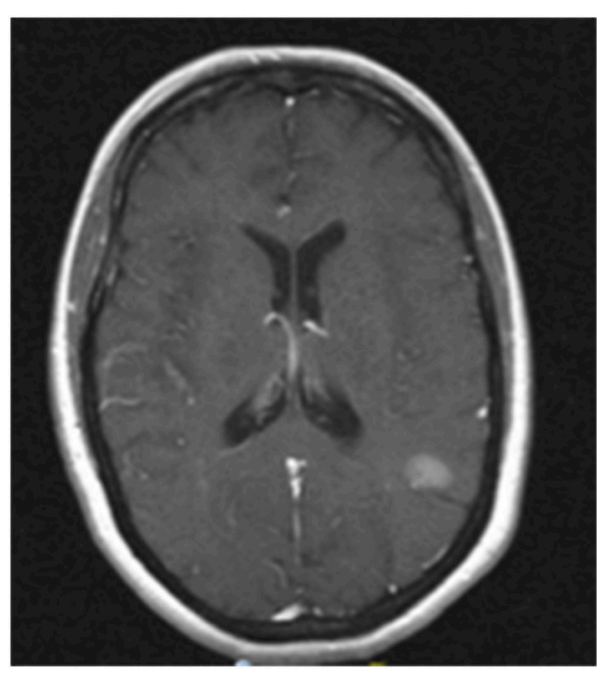

FIGURE 1 | MRI image of progression of left temporoparietal metastasis. Contrast enhanced T1 MRI showing a $1.6 \mathrm{~cm}$ left temporoparietal lesion that increased in size after completion of whole brain radiation. This lesion was treated subsequently with stereotactic radiosurgery.

once patients are diagnosed with melanoma brain metastasis $(2,3)$. Poor non-CNS disease control, poor performance status, leptomeningeal disease (LMD) defined as the infiltration of cancer cells in pia mater and arachnoid membrane, more than three CNS brain metastatic melanoma lesions, and a higher cumulative intracranial tumor volume have been associated with poor survival $(2,4,5)$. Surgery for melanoma brain metastasis with or without adjuvant whole brain radiation therapy has been shown to improve the median survival to $\sim 9$ months regardless whether patients have single or multiple brain metastases (3). However, depending on the location and the morbidity of surgery, this treatment option is sometimes not reasonable, especially in the setting of diffuse leptomeningeal involvement or periventricular ependymal involvement.

Ependymal spread is rare and is frequently associated with LMD. Flow of tumor cells in cerebrovascular fluid (CSF) through the ventricular system can seed the leptomeninges. Conversely, LMD along the surface of the brain and spinal cord may circulate through the CSF and deposit tumor cells along the ependymal surface leading to periventricular ependymal disease involvement. Ependymal disease is often clustered with LMD, although it is unclear whether differences exist in the underlying biology of and clinical outcomes between these two different presentations (6).

BRAFV600E mutation is observed in $40-50 \%$ of melanomas (7). The incidence of brain metastasis in BRAF-mutated or BRAF-wild type tumors is similar. However, a lower incidence of brain metastases was observed in patients with BRAFmutated tumors who received targeted therapy before the development of brain metastasis, suggesting that targeting BRAF may reduce metastatic spread to the brain or inhibit the growth of micrometastatic brain metastases (8). BRAF mutated melanoma brain metastases were shown to be more resistant to conventional treatment (including systemic agents alone, WBRT, or radiosurgery) when compared to BRAF wild 
A

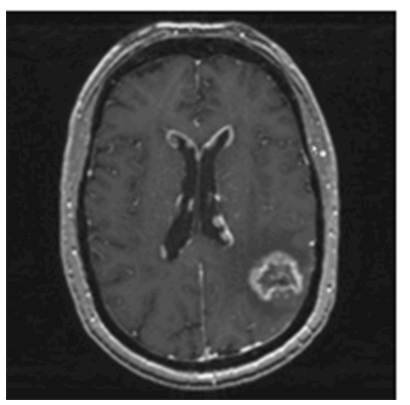

B

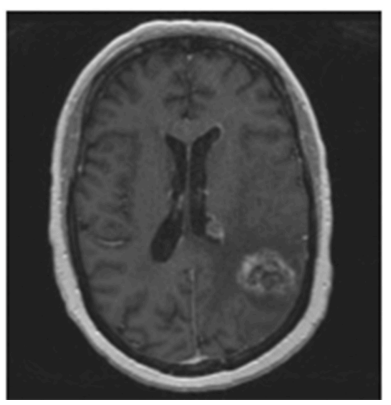

C

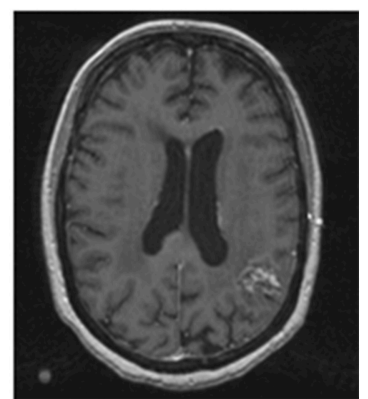

FIGURE 2 | MRI images of ependymal disease and response to whole brain re-irradiation. (A) Contrast enhanced T1 MRI showing new enhancing, linear, and nodular lesions along the ventricles consistent with ependymal disease. (B) Contrast enhanced T1 MRI performed 2 months after whole brain re-irradiation showing a significant and rapid decrease in ependymal enhancement. The treated metastasis in the left temporoparietal area was stable. (C) Contrast enhanced T1 MRI performed 1 year after whole brain radiation showing resolution of nodular lesions along the ventricles and no new brain metastases.

type metastases (HR $2.83 p=0.01$ ) (9). LMD is particularly associated with poor median survival of only 2 months despite treatment. Little is known about ependymal spread without LMD. Our patient with BRAFV600E mutant metastatic melanoma had extensive ependymal spread with no evidence of gross LMD. She survived for 28 months after initial diagnosis of brain metastasis and 11 months after diagnosis of significant ependymal disease.

\section{DISCUSSION}

Targeted therapy has modernized the treatment approach to BRAF-mutant metastatic melanoma. Vemurafenib was shown in a randomized controlled trial to prolong overall survival (10). Dabrafenib has been shown to improve progression-free survival (11). Single-agent BRAF inhibitors showed an excellent systemic response rate of $\sim 50 \%$. Response duration, however, was short lived with median of 6-7 months $(10,11)$. Both of these drugs are known to be effluxed by the intact blood brain barrier's p-glycoprotein hence have a poor CNS penetrance (12). Dabrafenib has been shown to have slightly better CNS penetration than vemurafenib (13). Clinically, the intracranial tumor response to vemurafenib in BRAF-mutation-positive melanoma patients was seen in seven out of 19 patients $(37 \%)$ (14). The intracranial response to dabrafenib ranges between 31 and $39 \%$ in patients with brain metastatic BRAF mutated melanoma from the BREAK -MB phase two trial (15). Addition of MEK inhibitors to dabrafenib can improve the rate and duration of response (16). Combination of BRAF and MEK inhibitors are now the standard of care for BRAF-mutated melanoma patients and have demonstrated some intracranial activity. Objective response rates are as high as $31 \%$ with dabrafenib in the BRAF mutant population $(17,18)$. Despite that, given the relatively poor $\mathrm{BBB}$ penetrance of anti-BRAF drugs, the brain remains a common site of treatment failure for BRAFtargeted therapy driving the poor outcome and survival of this patient population (19). Moreover, LMD has been traditionally approached by best supportive care and palliative therapy given its very poor outcome despite the multiple treatment modalities utilized (2).
However, in our case, we present a patient who survived 11 months after being diagnosed with significant ependymal disease. This was remarkable compared to a median survival of $8-10$ weeks from the literature $(2,20)$. Unfortunately, this patient ultimately succumbed to her disease and developed steroid dependent radiation necrosis along with associated side effects. Similar case reports reported long term survival outcome in patients with BRAF-mutated melanoma with LMD (21). Some case reports have demonstrated improvement in LMD after vemurafenib $(22,23)$. Interestingly, we noted that the longest median survival was attained in patients maintained on combination BRAF/MEK inhibitors after brain irradiation $(21,23)$. Combination treatment is needed as patients with symptomatic brain metastases need a rapid response to relieve symptoms which is attained by radiation. Maintenance BRAF/MEK inhibitors are needed to sustain the response. A recent report of 39 patients with melanoma with LMD showed mixed response to radiation and targeted therapy. The majority of patients had an extremely poor prognosis, as expected, however, targeted therapy helped to maintain CNS disease remission especially when used in combination with radiation therapy (24).

Our patient,despite having nodular ependymal disease, showed impressive brain control and overall survival after radiation and targeted therapy. Interestingly, she did not show evidence of gross disease involving her leptomeninges. Whether she had circulating tumor cells in her CSF is unclear. The course of disease in our patient suggests that ependymal disease does not necessarily lead to LMD. This study suggests that patients with ependymal disease in the absence of LMD may have a different natural history than those with LMD and therefore should be classified separately. Moreover, aggressive therapy with radiation and targeted therapy may offer relatively long-term disease control.

\section{CONCLUDING REMARKS}

Metastatic melanoma with spread to the ependymal lining or leptomeninges is associated with very poor outcomes. Ependymal disease does not necessarily lead to LMD and may have 
more favorable outcomes. Our patient with BRAF-mutant melanoma survived for 11 months after diagnosis of nodular ependymal spread. Aggressive multimodality treatment with radiation therapy and targeted therapy should be considered for these patients.

\section{ETHICS STATEMENT}

Written consent from the patient's next of kin was obtained for publication of the case report.

\section{REFERENCES}

1. Siegel RL, Miller KD, Jemal A. Cancer Statistics, 2017. CA. (2017) 67:7-30. doi: $10.3322 /$ caac. 21387

2. Davies MA, Liu P, McIntyre S, Kim KB, Papadopoulos N, Hwu WJ, et al. Prognostic factors for survival in melanoma patients with brain metastases. Cancer. (2011) 117:1687-96. doi: 10.1002/cncr.25634

3. Fife KM, Colman MH, Stevens GN, Firth IC, Moon D, Shannon KF, et al. Determinants of outcome in melanoma patients with cerebral metastases. $J$ Clin Oncol. (2004) 22:1293-300. doi: 10.1200/JCO.2004.08.140

4. Hirshman BR, Wilson BR, Ali MA, Schupper AJ, Proudfoot JA, Goetsch SJ, et al. Cumulative intracranial tumor volume augments the prognostic value of diagnosis-specific graded prognostic assessment model for survival in patients with melanoma cerebral metastases. Neurosurgery. (2018) 83:237-44. doi: 10.1093/neuros/nyx380

5. Raizer JJ, Hwu WJ, Panageas KS, Wilton A, Baldwin DE, Bailey E, et al. Brain and leptomeningeal metastases from cutaneous melanoma: survival outcomes based on clinical features. Neuro Oncol. (2008) 10:199-207. doi: 10.1215/15228517-2007-058

6. Debnam JM, Mayer RR, Chi TL, Ketonen L, Weinberg JS, Wei W, et al. Most common sites on MRI of intracranial neoplastic leptomeningeal disease. J Clin Neurosci. (2017) 45:252-6. doi: 10.1016/j.jocn.2017.07.020

7. Gray-Schopfer VC, da Rocha Dias S, Marais R. The role of B-RAF in melanoma. Cancer Meta Rev. (2005) 24:165-83. doi: 10.1007/s10555-005-5865-1

8. Gummadi T, Zhang BY, Valpione S, Kim C, Kottschade LA, Mittapalli RK, et al. Impact of BRAF mutation and BRAF inhibition on melanoma brain metastases. Melanoma Res. (2015) 25:75-9. doi: 10.1097/CMR.0000000000000133

9. Fang P, Boehling NS, Koay EJ, Bucheit AD, Jakob JA, Settle SH, et al. Melanoma brain metastases harboring BRAF (V600K) or NRAS mutations are associated with an increased local failure rate following conventional therapy. J Neuro Oncol. (2018) 137:67-75. doi: 10.1007/s11060-017-2695-2

10. Chapman PB, Hauschild A, Robert C, Haanen JB, Ascierto P, Larkin J, et al. Improved survival with vemurafenib in melanoma with BRAF V600E mutation. N Engl J Med. (2011) 364:2507-16. doi: 10.1056/NEJMoa1103782

11. Hauschild A, Grob JJ, Demidov LV, Jouary T, Gutzmer R, Millward M, et al. Dabrafenib in BRAF-mutated metastatic melanoma: a multicentre, open-label, phase 3 randomised controlled trial. Lancet. (2012) 380:358-65. doi: 10.1016/S0140-6736(12)60868-X

12. Mittapalli RK, Vaidhyanathan S, Sane R, Elmquist WF. Impact of Pglycoprotein $(\mathrm{ABCB} 1)$ and breast cancer resistance protein (ABCG2) on the brain distribution of a novel BRAF inhibitor: vemurafenib (PLX4032). J Pharmacol Exp Ther. (2012) 342:33-40. doi: 10.1124/jpet.112.192195

13. Mittapalli RK, Vaidhyanathan S, Dudek AZ, Elmquist WF. Mechanisms limiting distribution of the threonine-protein kinase B-RaF(V600E) inhibitor dabrafenib to the brain: implications for the treatment of melanoma brain metastases. J Pharmacol Exp Ther. (2013) 344:655-64. doi: 10.1124/jpet.112.201475

14. Dummer R, Goldinger SM, Turtschi CP, Eggmann NB, Michielin O, Mitchell $\mathrm{L}$, et al. Vemurafenib in patients with BRAF(V600) mutation-positive melanoma with symptomatic brain metastases: final results of an open-label pilot study. Eur J Cancer. (2014) 50:611-21. doi: 10.1016/j.ejca.2013.11.002

\section{AUTHOR CONTRIBUTIONS}

IA-G summarizing the case and writing the paper. GHS, SC, EM, JS, AM, and MM reviewing and editing manuscript. JY revising, editing, and coordinating the manuscript.

\section{FUNDING}

This study is supported by the Cleveland Clinic.

15. Kirkwood JM, Long GV, Trefzer U, Davies MA, Ascierto PA, Chapman $\mathrm{PB}$, et al. BREAK-MB: a phase II study assessing overall intracranial response rate (OIRR) to dabrafenib (GSK2118436) in patients (pts) with BRAF V600E/k mutation-positive melanoma with brain metastases (mets). J Clin Oncol. (2012) 30 (15 Suppl.):8501. doi: 10.1200/jco.2012.30.15_ suppl.8501

16. Flaherty KT, Infante JR, Daud A, Gonzalez R, Kefford RF, Sosman J, et al. Combined BRAF and MEK inhibition in melanoma with BRAF V600 mutations. N Engl J Med. (2012) 367:1694-703. doi: 10.1056/NEJMoa 1210093

17. Long GV, Stroyakovskiy D, Gogas H, Levchenko E, de Braud F, Larkin J, et al. Combined BRAF and MEK inhibition versus BRAF inhibition alone in melanoma. N Engl J Med. (2014) 371:1877-88. doi: 10.1056/NEJMoa 1406037

18. Long GV, Trefzer U, Davies MA, Kefford RF, Ascierto PA, Chapman PB, et al. Dabrafenib in patients with Val600Glu or Val600Lys BRAF-mutant melanoma metastatic to the brain (BREAK-MB): a multicentre, open-label, phase 2 trial. Lancet Oncol. (2012) 13:1087-95. doi: 10.1016/S1470-2045(12)70431-X

19. Sloot S, Chen YA, Zhao X, Weber JL, Benedict JJ, Mule JJ, et al. Improved survival of patients with melanoma brain metastases in the era of targeted BRAF and immune checkpoint therapies. Cancer. (2018) 124:297-305. doi: $10.1002 / \mathrm{cncr} .30946$

20. Harstad L, Hess KR, Groves MD. Prognostic factors and outcomes in patients with leptomeningeal melanomatosis. Neuro Oncol. (2008) 10:1010-8. doi: 10.1215/15228517-2008-062

21. Kim DW, Barcena E, Mehta UN, Rohlfs ML, Kumar AJ, Penas-Prado M, et al. Prolonged survival of a patient with metastatic leptomeningeal melanoma treated with BRAF inhibition-based therapy: a case report. BMC Cancer. (2015) 15:400. doi: 10.1186/s12885-015-1391-x

22. Schafer N, Scheffler B, Stuplich M, Schaub C, Kebir S, Rehkamper C, et al. Vemurafenib for leptomeningeal melanomatosis. J Clin Oncol. (2013) 31:e173-4. doi: 10.1200/JCO.2012.46.5773

23. Lee JM, Mehta UN, Dsouza LH, Guadagnolo BA, Sanders DL, Kim KB. Long-term stabilization of leptomeningeal disease with wholebrain radiation therapy in a patient with metastatic melanoma treated with vemurafenib: a case report. Melan Res. (2013) 23:175-8. doi: 10.1097/CMR.0b013e32835e589c

24. Geukes Foppen MH, Brandsma D, Blank CU, van Thienen JV, Haanen JB, Boogerd W. Targeted treatment and immunotherapy in leptomeningeal metastases from melanoma. Ann Oncol. (2016) 27:1138-42. doi: 10.1093/annonc/mdw134

Conflict of Interest Statement: The authors declare that the research was conducted in the absence of any commercial or financial relationships that could be construed as a potential conflict of interest.

Copyright (C) 2019 Abu-Gheida, Chao, Murphy, Suh, Stevens, Mohammadi, McNamara and Yu. This is an open-access article distributed under the terms of the Creative Commons Attribution License (CC BY). The use, distribution or reproduction in other forums is permitted, provided the original author(s) and the copyright owner(s) are credited and that the original publication in this journal is cited, in accordance with accepted academic practice. No use, distribution or reproduction is permitted which does not comply with these terms. 